\title{
The Enigma of AA Dor
}

\author{
Thomas Rauch \\ Dr.-Remeis-Sternwarte, D-96049 Bamberg, Germany \\ Institut für Astronomie und Astrophysik, D-72076 Tübingen, Germany \\ Klaus Werner \\ Institut für Astronomie und Astrophysik, D-72076 Tübingen, Germany
}

\begin{abstract}
AA Dor (LB 3459) is an eclipsing, close binary $(P=0.26 \mathrm{~d})$ consisting of a sdOB primary star and an unseen secondary with an extraordinary small mass. The secondary is possibly a former planet which may have survived a common-envelope phase and has even gained mass.

In order to investigate on an existing discrepancy between the components' mass derived from NLTE spectral analysis and subsequent comparison to evolutionary tracks and masses derived from radial-velocity and the eclipse curves, we performed phase-resolved high-resolution and high-SN spectroscopy with the UVES attached to the ESO VLT. From the obtained spectra, we have determined AA Dor's orbital parameters $(P=22600.702 \pm 0.005 \mathrm{sec}, A=39.19 \pm 0.05$ $\left.\mathrm{km} \mathrm{s}^{-1}, T_{0}=2451917.152690\right)$ and the rotational velocity $\left(v_{\text {rot }}=47 \pm 5 \mathrm{~km} \mathrm{~s}^{-1}\right)$ of the primary.
\end{abstract}

Hilditch et al. (1996) have analyzed that the primary component of AA Dor has a mass of $M_{1}=0.5 \mathrm{M}_{\odot}$ and the cool secondary has a very small mass of $M_{2}=0.086 \mathrm{M}_{\odot}$. The latter is in excellent agreement with lowest mass ZAMS models of Dorman et al. (1989). In a recent spectral analysis (Rauch 2000) of the primary, based on high-resolution CASPEC and IUE spectra, $M_{1}=0.330$ $\mathrm{M}_{\odot}$ has been derived from comparison of $T_{\text {eff }}$ and $\log g$ to evolutionary tracks of post-RGB stars (Driebe et al. 1998) and $M_{2}=0.066 \mathrm{M}_{\odot}$ has subsequently been calculated from the system's mass function. Thus, the secondary is possibly a planet which may have survived a common envelope phase and has even gained mass ("late case B mass transfer", Iben \& Livio 1993). However, a reason for the discrepancy between these masses and those derived from the radial-velocity and the eclipse curves (Kilkenny et al. 1979) is not known. Two possible reasons are the inaccuracy of the theoretical models by Driebe et al. (1998) for AA Dor and the error range for the photospheric parameter determination in Rauch (2000). This analysis was hampered by the long exposure times (some hours) and hence, a large orbital velocity coverage.

In order to make progress and to minimize the effects of orbital motion, 105 UVES (attached to the ESO VLT) spectra had been taken on Jan 8, 2001 with an exposure time of $180 \mathrm{sec}$ each. We achieved a resolving power of 48000 at an average $S / N \approx 20$. The spectra cover a complete orbital period of AA Dor.

The phase-dependent radial velocity is determined by fitting Lorentzians to the sharpest line in the optical spectrum of AA Dor, He II $\lambda 4686 \AA$ (Rauch 2000). For this procedure the smearing due to orbital motion during the exposure was 


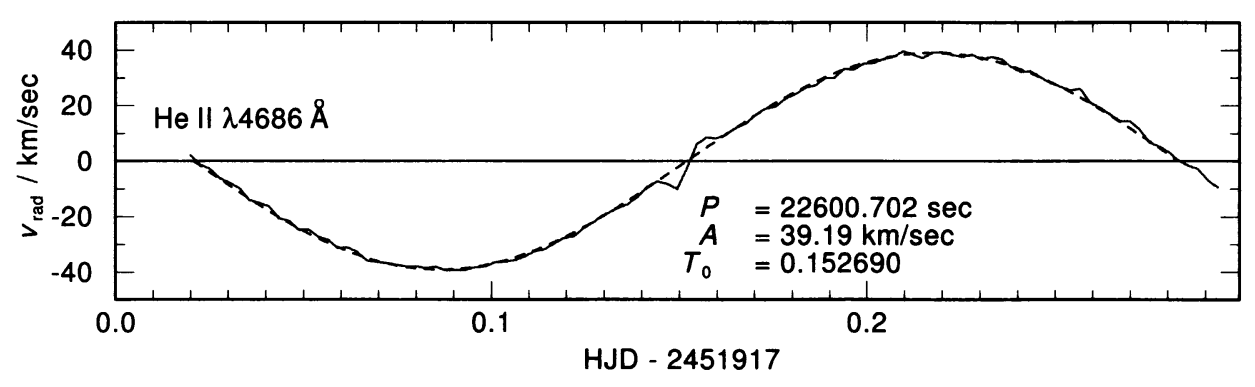

Figure 1. Radial-velocity curve of AA Dor measured from He II $\lambda 4686 \AA$ compared to a sine curve (dashed). Note the velocity jumps close to $T_{0}$ which are the result of the transit of the cool companion (Rossiter Effect, Rossiter 1924).

neglected because the exposure time is very short compared to the complete period. Then, the derived velocity curve was fitted by a sine curve (Fig. 1). We adopt $P=22600.702 \mathrm{sec}$ and $A=39.19 \mathrm{~km} \mathrm{~s}^{-1}$. The period is by $0.02 \%$ longer than that given by Kilkenny et al. 1991 (22597.0sec from photometric data of 27 eclipses). A higher radial-velocity amplitude of $A=40.8 \mathrm{~km} \mathrm{~s}^{-1}$ has been measured by Hilditch et al. (1996), also from observations of He II $\lambda 4686 \AA$.

We calculated a grid of $\mathrm{H}+\mathrm{He}$ composed NLTE model atmospheres and performed a $\chi^{2}$ test (wavelength, flux level, $\mathrm{He} / \mathrm{H}$, and $v_{\text {rot }}$ ) in order to determine the rotational velocity of the primary by comparison to the co-added UVES spectrum. We keep $T_{\text {eff }}=42 \mathrm{kK}$ and $\log g=5.2(\mathrm{cgs})$ fixed (Rauch 2000) and achieve the best fit at $v_{\text {rot }}=47 \mathrm{~km} \mathrm{~s}^{-1}$ and $\mathrm{He} / \mathrm{H}=0.0008$ (by number).

The determined rotational velocity of the primary of $v_{\text {rot }}=47 \mathrm{~km} \mathrm{~s}^{-1}$ suggests, that AA Dor performs a bound rotation $\left(45.7 \mathrm{~km} \mathrm{~s}^{-1}\right)$. The components' mass determined by Rauch (2000) are verified. The cool component has almost the same radius like Jupiter but its mass is about $1 / 15 \mathrm{M}_{\odot}$ which is 70 times higher than Jupiter's mass. Thus, it lies formally within the brown-dwarf mass range $\left(0.013-0.08 \mathrm{M}_{\odot}\right)$. However, the idea of Rauch (2000), that it may have been a planet which has survived the common envelope phase and even has gained mass, needs further numerical simulations of the common envelope phase to be verified.

This research was supported by the DLR (grants 50 OR 97055 and 50 OR 0201).

\section{References}

Dorman B., Nelson L.A., Chau W.Y. 1989, ApJ 342, 1003

Kilkenny D., Harrop-Allin M., Marang F. 1991, Inf. Bull. on Variable Stars, No. 3569

Hilditch R.W., Harries T.J., Hill G. 1996, MNRAS 279, 1380

Iben I.Jr., Livio M. 1993, PASP 105, 1373

Rauch T. 2000, A\&A 356, 665

Rossiter, R.A., 1924, ApJ 60, 15 